\title{
FUNCTIONAL DECOMPOSITION OF ECOLOGICAL NETWORKS BY PROBABILITY METHOD
}

\section{YANG LIU}

Shenzhen Technology University

Shenzhen

P. R. China

e-mail: liuyang2@sztu.edu.cn

\begin{abstract}
In this paper, we mainly study the functional decomposition of ecological networks or systems, involving both chains and cycles, from the perspective of probability. We establish some results on the probability and expectation of on the chains and cycles in a decomposition for a complex network system, and furthermore, we obtain the coefficients in functional decompositions.
\end{abstract}

\section{Introduction}

Complex ecological systems or networks describe the relationships of organisms in a biosphere, which can be dynamic (see, for instance, [2] and [12]). There have been many research works on the structure of ecological systems, the energy transfers, and the dynamics of ecological systems (see, for instance, [5], [10], and [6]). Recently, there have been extensive studies on the dimensionality of ecological networks (see, for example, [4],

2020 Mathematics Subject Classification: 92F05, 93B11, 91B74.

Keywords and phrases: models of real-world systems, system structure, probability method, ecological networks, chains.

Received March 28, 2020; Revised May 18, 2020

(C) 2020 Scientific Advances Publishers 
[11], and [7]). The probability method has recently been used to the study of the social structure of a given population (see, for instance, [9] and [1]), but in this paper, we will use probability method to do functional decompositions for complex ecological network systems.

A complex ecological system or networks can be decomposed into simple chains and cycles, and in this paper, we show the functional decomposition of ecological networks, involving both chains and cycles, from the perspective of probability. This paper is structure as follows: in Section 2, we work on the null space of flux matrix. In Section 3, we introduce some notations and approach in functional decompositions for complex ecological network systems. In Section 4, we establish theorems on the probability and expectation of on the chains and cycles in a decomposition for a complex network system, and furthermore, we obtain the coefficients in functional decompositions.

\section{Null Space of Flux Matrix}

The dimension of the null space of the flux matrix can be obtained from the topology of the network system, the number of compartments and the number of flows. By running a simulation model, we can get the coefficients for the fluxes. We can also compute it by Mathematica. For instance, for the model considered in the graph of a network system in Figure 2.1, we have

$$
D=\left(\begin{array}{llllll}
1 & 1 & 0 & 0 & 0 & 0 \\
0 & 0 & 1 & 0 & 0 & 1 \\
0 & 1 & 1 & 0 & 0 & 0 \\
1 & 0 & 0 & 0 & 0 & 1 \\
1 & 1 & 0 & 1 & 1 & 0 \\
0 & 0 & 0 & 1 & 0 & 0 \\
0 & 0 & 0 & 0 & 1 & 0 \\
1 & 0 & 0 & 0 & 1 & 1
\end{array}\right),
$$


and

$$
F=\left(\begin{array}{c}
0.125 \\
0.2 \\
0.225 \\
0.1 \\
0.325 \\
0.15 \\
0.05 \\
0.15
\end{array}\right) .
$$

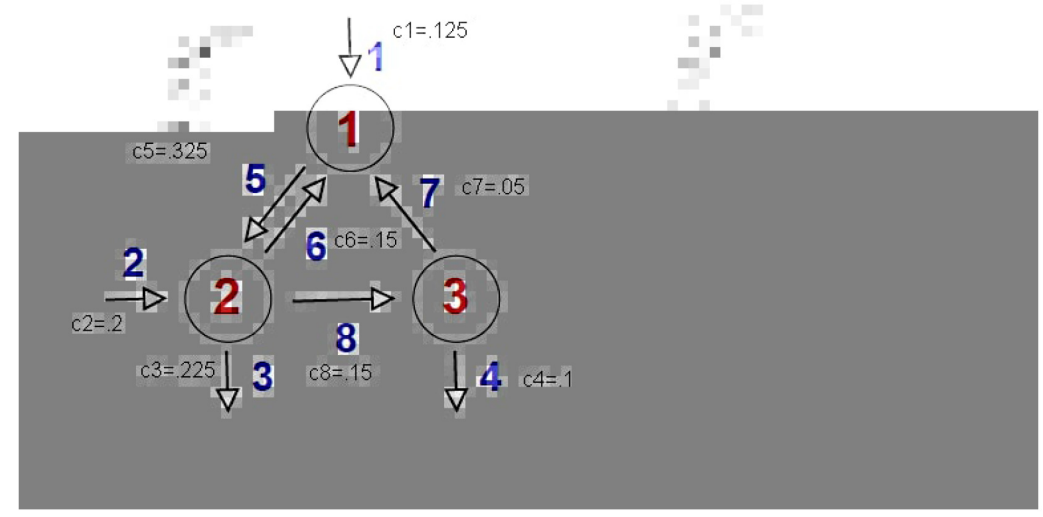

Figure 2.1. Graph of a network system.

Using the linear solver in mathematics, we can get some solutions, and the dimension of the null space by compute the rank of the matrix using Mathematica as well. It has an advantage in computation over using the topology of the system, and moreover, it is good at symbolic computations as well as numerical computations, which gives the exact dimension of the null space. 


\section{Introduction on Functional Decomposition}

Now let's consider a network system $\mathcal{S}$ and list some notations for the system that was used in [8] and we are going to use later on.

$C_{i}: \quad$ Compartment $i$;

$F_{i j}: \quad$ Flow from compartment $j$ to compartment $i$ per unit of time;

$z_{i}: \quad$ Input from the environment to $i$

$y_{i}: \quad$ Output to the environment from compartment $i$;

$T_{i}^{\text {in }}: \quad$ throughput (sum of inflows) into compartment $i$;

$T_{i}^{\text {out }}: \quad$ throughflow (sum of outflows) out of compartment $i$.

We say a flux $F_{i}$ intersects $F_{j}$ if they share at least one compartment. Otherwise, we say their intersection is empty.

For any given complex network system, algorithms that can be easily developed to identify all the simple chains and cycles, and furthermore, one can count the number of chains in the decomposition.

\section{Probability and Expectation}

Moreover, the probability that a particle goes through a basic pathway can be computed directly from the flows as follows. The coefficients of the simple chains were computed by solving linear systems, and therefore, in general, we have

Proposition 1. Let $C_{1}, C_{2}, \cdots, C_{n}$ be the compartments of a network system $\mathcal{S}, \mathcal{F}$ be a chain containing compartments $C_{n_{0}}$ with input from the environment $z_{n_{0}}>0$ and $C_{n_{1}}, C_{n_{2}}, \cdots, C_{n_{k}}$ with no inputs from the environment and $F_{n_{j}}^{\text {out }}$ be the flow out of compartment $C_{n_{j}}, j=1,2, \ldots, k$ in $\mathcal{F}$, and $\mathbb{P}(\mathcal{F})$ be the probability that a particle goes through $\mathcal{F}$. Then 


$$
\mathbb{P}(\mathcal{F})=\frac{z_{n_{0}}}{\sum_{i=1}^{n} z_{i}} \prod_{j=1}^{k} \frac{F_{n_{j}}^{\text {out }}}{T_{n_{j}}^{\text {out }}}
$$

For the cycles, the probabilities can be also derived.

Theorem 2. Let $\mathcal{C}$ be a cycle containing compartments $C_{m_{1}}, C_{m_{2}}, \cdots, C_{m_{k}}$ and $F_{n_{j}}^{\text {out }}$ be the flow out of compartment $C_{m_{j}}, j=1,2, \ldots, k$ in $\mathcal{F}$, and $\mathbb{P}(\mathcal{C})$ be the probability that a particle goes through $\mathcal{C}$. Then

$$
\mathbb{P}(\mathcal{C})=\prod_{j=1}^{k} \frac{F_{m_{j}}^{\text {out }}}{T_{m_{j}}^{\text {out }}}
$$

Proof. For any particle that enters the system, the probability that it goes out of the compartment $C_{m_{j}}$ is $\frac{F_{m_{j}}^{\text {out }}}{T_{m_{j}}^{\text {out }}}$. By conditional probability, we obtain the probability that the particle goes through $\mathcal{C}$ is $\prod_{j=1}^{k} \frac{F_{m_{j}}^{\text {out }}}{T_{m_{j}}^{\text {out }}}$.

This completes the proof.

Let's see a simple system with integer flow rates.

Example 3. The following simple system (called "simple toy" model) can be decomposed by using the probability as

Definition 4. Let $F$ be a flux, $L$ be a random pathway, $\mathbb{P}(L)$ is the probability of pathway $L$ occurring, $n(L, F)$ is the number of times flux $F$ appearing in pathway $L$. Then we can define the Expect Time of this flux appearing on a pathway by,

$$
\mathbb{E}(F):=\sum_{L \text { is any path }} n(L, F) \mathbb{P}(L),
$$


in which $\mathbb{P}(L)$ is the product of flow probabilities that make up the pathway, $\mathbb{E}(F)$ is a sum over all pathways. It could be an infinite sum, and $n(L, F)$ can be larger than one only if $F$ is a cycle.
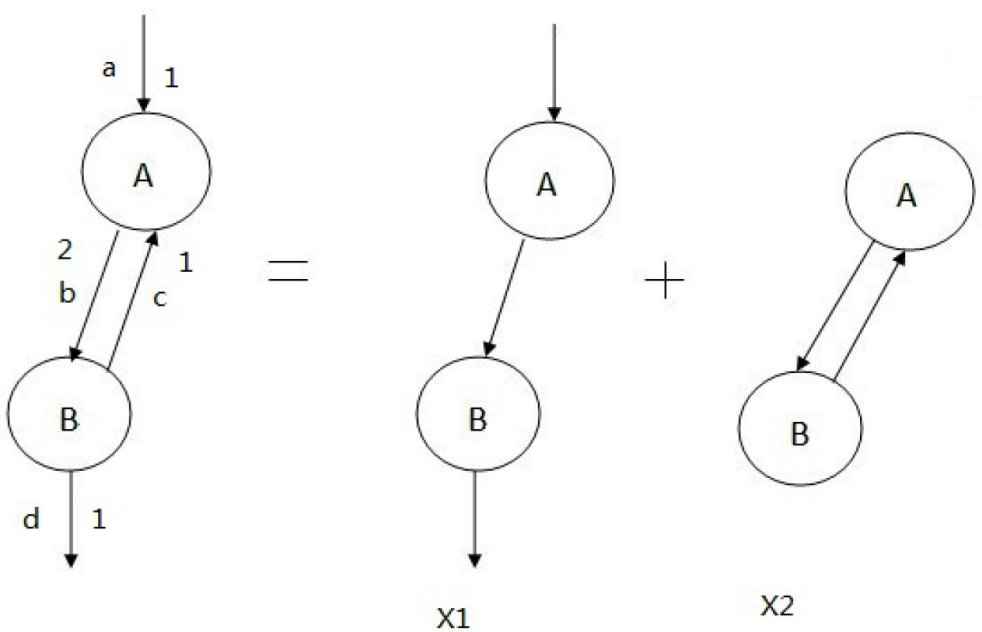

Figure 4.1. "Simple toy" model.
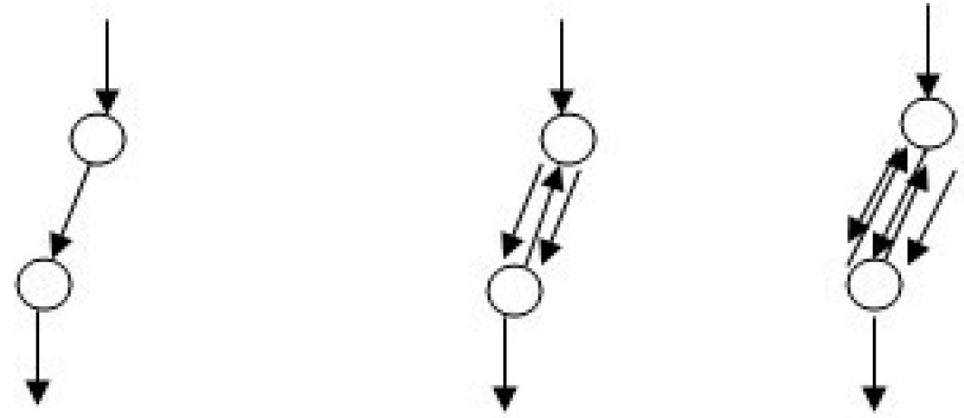

Figure 4.2. 
Example 5 (Simple toy model (Figure 4.1)). The probabilities of flows $a, b, c, d$ are: $1,1, \frac{1}{2}, \frac{1}{2}$. The probabilities of fluxes $X_{1}, X_{2}$ are: $\frac{1}{2}, \frac{1}{2} ; X_{1}$ is a chain, so $n\left(L, X_{1}\right) \leq 1$. We only care about when $n\left(L, X_{1}\right)_{1}=1$, which means $X_{1}$ occurs only once.

The probability of each case: $\frac{1}{2}, \frac{1}{2} \times \frac{1}{2}, \frac{1}{2} \times\left(\frac{1}{2}\right)^{2} \ldots$. So, we can compute

$$
\mathbb{E}\left(X_{1}\right)=1 \times\left[\sum_{n=1}^{\infty}\left(\frac{1}{2}\right)^{n}\right]=1
$$

$X_{2}$ is a cycle, so it can appear infinitely many times in a pathway.

Base case: $X_{2}$ appears only once in a pathway,

The probability for this is $\frac{1}{4}$;

$X_{2}$ appears twice in a pathway,

The probability for this is $\frac{1}{8}$.

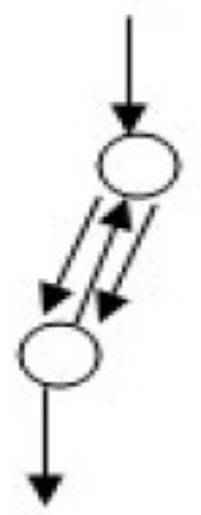

Figure 4.3. 


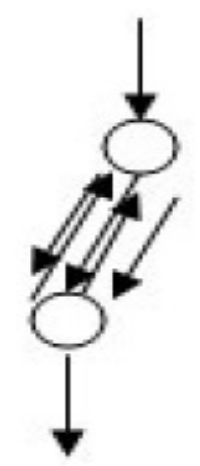

Figure 4.4.

Thus we can use induction to obtain that the probability of $X_{2}$ appears $n$ times in a pathway is $\frac{1}{2} \times\left(\frac{1}{2}\right)^{n}$ and the expectation is

$$
\mathbb{E}\left(X_{2}\right)=\sum_{n=1}^{\infty} \frac{n}{2^{n+1}}=1 .
$$

In general, we have the following theorem regarding the expectations on the chains and cycles in a decomposition for a complex network system.

Theorem 6. Let $\mathcal{F}_{1}, \mathcal{F}_{2}, \ldots, \mathcal{F}_{n}$ be the chains and $\mathcal{C}_{1}, \mathcal{C}_{2}, \ldots, \mathcal{C}_{m}$ be the cycles in the decomposition. The expected times that the chains

$$
\mathbb{E}\left(\mathcal{F}_{i}\right)=\frac{\mathbb{P}\left(\mathcal{F}_{i}\right)}{1-\sum_{\mathcal{C}_{j} \cap \mathcal{F}_{i} \neq \emptyset} \mathbb{P}\left(\mathcal{C}_{j}\right)},
$$

for all $i$, and

$$
\mathbb{E}\left(\mathcal{C}_{i}\right)=\frac{\mathbb{P}\left(\mathcal{C}_{i}\right) \sum_{\mathcal{C}_{j} \cap \mathcal{F}_{i} \neq \emptyset} \mathbb{P}\left(\mathcal{F}_{i}\right)}{\left(1-\sum_{\mathcal{C}_{j} \cap \mathcal{C}_{i} \neq \emptyset} \mathbb{P}\left(\mathcal{C}_{j}\right)\right)^{2}},
$$

for all $i$. 
Proof. It is not hard to see that (4.5) holds.

Let $p_{j}:=\mathbb{P}\left(\mathcal{C}_{j}\right)$ and $\left\{j_{1}, \cdots, j_{k}\right\}:=\left\{j: \mathcal{C}_{j} \cap \mathcal{C}_{i} \neq \emptyset\right\}$. Then we have

$$
\begin{aligned}
\frac{\mathbb{E}\left(\mathcal{C}_{i}\right)}{\sum_{\mathcal{C}_{j} \cap \mathcal{F}_{i} \neq \emptyset} \mathbb{P}\left(\mathcal{F}_{i}\right)} & =\sum_{l=0}^{\infty} l p_{i}^{l} \sum_{l_{j_{1}}, \cdots, l_{j_{k}}=0}^{\infty}\left(\begin{array}{c}
l+l_{j_{1}}+\cdots+l_{j_{k}} \\
l
\end{array}\right) p_{j_{1}}^{l_{j_{1}}} \ldots p_{j_{k}}^{l_{j_{k}}} \\
& =: \quad f\left(p_{i}\right) .
\end{aligned}
$$

Divided by $p_{i}$, (4.7) becomes

$$
\frac{f\left(p_{i}\right)}{p_{i}}=\sum_{l=1}^{\infty} l p_{i}^{l-1} \sum_{l_{j_{1}}, \cdots, l_{j_{k}}=0}^{\infty}\left(\begin{array}{c}
l+l_{j_{1}}+\cdots+l_{j_{k}} \\
l
\end{array}\right) p_{j_{1}}^{l_{j_{1}}} \cdots p_{j_{k}}^{l_{j_{k}}} .
$$

Then taking the integral, we have

$$
\begin{aligned}
\int \frac{f\left(p_{i}\right)}{p_{i}} d p_{i} & =\sum_{l, l_{j_{1}}, \cdots, l_{j_{k}}=0}^{\infty}\left(\begin{array}{c}
l+l_{j_{1}}+\cdots+l_{j_{k}} \\
l
\end{array}\right) p_{i}^{l} p_{j_{1}}^{l_{j_{1}}} \cdots p_{j_{k}}^{l_{j_{k}}} \\
& =\frac{1}{\left(1-p_{i}-p_{j_{1}}-\cdots-p_{j_{k}}\right)} .
\end{aligned}
$$

To get $f\left(p_{i}\right)$, we take the derivative of (4.9) with respect to $p_{i}$ and then multiply it by $p_{i}$. Thus we get

$$
f\left(p_{i}\right)=\frac{p_{i}}{\left(1-p_{i}-p_{j_{1}}-\cdots-p_{j_{k}}\right)^{2}},
$$

and then (4.6) follows.

Now, we can do the functional decomposition for system $\mathcal{S}$.

Theorem 7. Let $\mathcal{F}_{1}, \mathcal{F}_{2}, \ldots, \mathcal{F}_{n}$ be the chains and $\mathcal{C}_{1}, \mathcal{C}_{2}, \ldots, \mathcal{C}_{m}$ be the cycles in the decomposition. Then in the functional decomposition, the coefficient of $\mathcal{F}_{i}$,

$$
c_{i}=\mathbb{E}\left(\mathcal{F}_{i}\right) \sum_{i=1}^{n} z_{i}
$$


for $i=1,2, \cdots, n$ and the coefficient of $\mathcal{C}_{j}$,

$$
d_{j}=\mathbb{E}\left(\mathcal{C}_{j}\right) \sum_{i=1}^{n} z_{i}
$$

for $j=1,2, \cdots, m$.

Proof. Since the sum of the inputs from the environment to the system $\sum_{i=1}^{n} z_{i}$ is decomposed in terms of the proportions, $\mathbb{E}\left(\mathcal{F}_{i}\right)$ and $\mathbb{E}\left(\mathcal{C}_{j}\right)$, the coefficients follow.

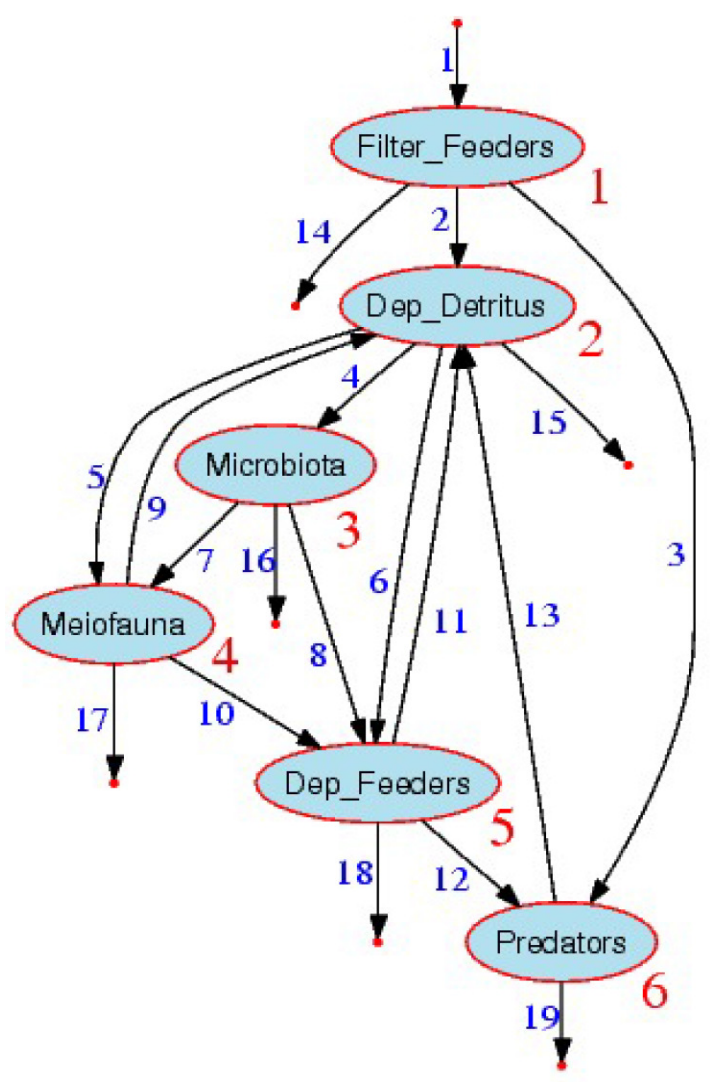

Figure 4.5. Oyster-reef model. 
In particular, we can apply the above theorem to do the functional decomposition for the oyster-reef model (see [3]), of which the diagram is shown in Figure 4.5.

\section{Acknowledgement}

This work is partially supported by the Research Funding of Shenzhen Municipal Finance. The author would also like to thank the referee for the comments that have helped greatly improve the presentation of the work in this paper. In addition, the author would also like to thank Professor C. Kazanci for some discussions.

\section{References}

[1] Walid Ahmad, Mason A. Porter and Mariano Beguerisse-Díaz, Tie-decay temporal networks in continuous time and eigenvector-based centralities, arXiv preprint arXiv:1805.00193, 2018.

[2] Madhur Anand, Andrew Gonzalez, Frédéric Guichard, Jurek Kolasa and Lael Parrott, Ecological systems as complex systems: Challenges for an emerging science, Diversity 2(3) (2010), 395-410.

DOI: https://doi.org/10.3390/d2030395

[3] Richard F. Dame and Sergej Olenin, The Comparative Roles of Suspension-Feeders in Ecosystems: Proceedings of the NATO Advanced Research Workshop on The Comparative Roles of Suspension-Feeders in Ecosystems, Nida, Lithuania, 4-9 October 2003, Volume 47, Springer Science \& Business Media, 2005.

DOI: https://doi.org/10.1007/1-4020-3030-4

[4] Anna Eklöf, Ute Jacob, Jason Kopp, Jordi Bosch, Rocío Castro-Urgal, Natacha P. Chacoff, Bo Dalsgaard, Claudio de Sassi, Mauro Galetti, Paulo R. Guimarães, Silvia Beatriz Lomáscolo, Ana M. Martín González, Marco Aurelio Pizo, Romina Rader, Anselm Rodrigo, Jason M. Tylianakis, Diego P. Vázquez and Stefano Allesina, The dimensionality of ecological networks, Ecology Letters 16(5) (2013), 577-583.

DOI: https://doi.org/10.1111/ele.12081

[5] Crawford S. Holling, Resilience and stability of ecological systems, Annual Review of Ecology and Systematics 4(1) (1973), 1-23.

DOI: https://doi.org/10.1146/annurev.es.04.110173.000245

[6] Alf Hornborg, Towards an ecological theory of unequal exchange: Articulating world system theory and ecological economics, Ecological Economics 25(1) (1998), 127-136.

DOI: https://doi.org/10.1016/S0921-8009(97)00100-6 
[7] Pedro Jordano, Sampling networks of ecological interactions, Functional Ecology 30(12) (2016), 1883-1893.

DOI: https://doi.org/10.1111/1365-2435.12763

[8] L. Matamba, Caner Kazanci, J. R. Schramski, M. Blessing, P. Alexander and B. C. Patten, Throughflow analysis: A stochastic approach, Ecological Modelling 220(22) (2009), 3174-3181.

DOI: https://doi.org/10.1016/j.ecolmodel.2009.07.001

[9] Ioannis Psorakis, Probabilistic Inference in Ecological Networks; Graph Discovery, Community Detection and Modelling Dynamic Sociality, Ph.D. Thesis, Oxford University, UK, 2013.

[10] Peter Turchin and Andrew D. Taylor, Complex dynamics in ecological time series, Ecology 73(1) (1992), 289-305.

DOI: https://doi.org/10.2307/1938740

[11] Jason M. Tylianakis and Rebecca J. Morris, Ecological networks across environmental gradients, Annual Review of Ecology, Evolution, and Systematics 48 (2017), 25-48.

DOI: https://doi.org/10.1146/annurev-ecolsys-110316-022821

[12] Jianguo $\mathrm{Wu}$ and John L. David, A spatially explicit hierarchical approach to modeling complex ecological systems: Theory and applications, Ecological Modelling 153(1-2) (2002), 7-26.

DOI: https://doi.org/10.1016/S0304-3800(01)00499-9 